\title{
Time-resolving the UV-initiated photodissociation dynamics of OCS
}

Evangelos T. Karamatskos, ${ }^{1,2}$ Suresh Yarlagadda, ${ }^{3}$ Serguei Patchkovskii, ${ }^{3}$

Marc J. J. Vrakking, ${ }^{3}$ Ralph Welsch, ${ }^{1}$ Jochen Küpper, ${ }^{1,2,4, a)}$ and Arnaud Rouzée ${ }^{3, \text { b) }}$

1) Center for Free-Electron Laser Science, Deutsches Elektronen-Synchrotron DESY, Notkestraße 85, 22607 Hamburg, Germany

2) Department of Physics, Universität Hamburg, Luruper Chaussee 149, 22761

Hamburg, Germany

3) Max Born Institute, Max-Born-Straße 2a, 12489 Berlin, Germany

4) Center for Ultrafast Imaging, Universität Hamburg, Luruper Chaussee 149, 22761 Hamburg, Germany

(Dated: 2020-10-30)

We present a time-resolved study of the photodissociation dynamics of OCS after UVphotoexcitation at $\lambda=237 \mathrm{~nm}$. OCS molecules $\left(X^{1} \Sigma^{+}\right)$were primarily excited to the $1^{1} A^{\prime \prime}$ and the $2^{1} A^{\prime}$ Renner-Teller components of the ${ }^{1} \Sigma^{-}$and ${ }^{1} \Delta$ states. Dissociation into $\mathrm{CO}$ and $\mathrm{S}$ fragments was observed through time-delayed strong-field ionisation and imaging of the kinetic energy of the resulting $\mathrm{CO}^{+}$and $\mathrm{S}^{+}$fragments by intense $790 \mathrm{~nm}$ laser pulses. Surprisingly, fast oscillations with a period of $\sim 100$ fs were observed in the $\mathrm{S}^{+}$channel of the UV dissociation. Based on wavepacket-dynamics simulations coupled with a simple electrostatic-interaction model, these oscillations do not correspond to the known highly-excited rotational motion of the leaving $\mathrm{CO}\left(X^{1} \Sigma^{+}, J \gg 0\right)$ fragments, which has a timescale of $\sim 140$ fs. Instead, we suggest to assign the observed oscillations to the excitation of vibrational wavepackets in the $2{ }^{3} A^{\prime \prime}$ or $2{ }^{1} A^{\prime \prime}$ states of the molecule that predissociate to form $\mathrm{S}\left({ }^{3} P_{J}\right)$ photoproducts.

a jochen.kuepper@cfel.de https://www.controlled-molecule-imaging.org

b) arnaud.rouzee@mbi-berlin.de 


\section{INTRODUCTION}

Imaging ultrafast photochemical reactions with quantum-mechanical detail and atomic temporal and spatial resolution is one of the ultimate dreams of current physical chemistry and the molecular sciences in general. ${ }^{1-3}$ Various approaches have been discussed, ranging from $x-$ ray $^{3-6}$ and electron diffractive imaging ${ }^{78}$ to laser-induced electron diffraction(LIED) $)^{9} 10$ and Coulomb-explosion ion imaging. ${ }^{11-13}$ Complementary methods such as high-harmonic spectroscopy ${ }^{14-16}$ and time-resolved photoelectron spectroscopy ${ }^{17-19}$ have provided further insight into these processes. While the experimental recording of the envisioned highresolution "molecular movies" are still outstanding, promising examples demonstrate the progress that has been made, for instance, using time-resolved electron diffraction. ${ }^{20-22}$ We are interested in exploring the possibilities for atomically resolved imaging of photoinduced chemical reactions using LIED. ${ }^{23-25}$ In this paper, we present ion-imaging measurements that we have performed as the precursor to such a LIED experiment.

The carbonyl sulfide (OCS) molecule provides an interesting benchmark system and serves as an important testbed for the study of photodissociation dynamics. OCS is linear in its rovibronic ground state $\left(X^{1} \Sigma^{+}\right)$, but it bends in its first absorption band upon photoexcitation in the range from $190 \mathrm{~nm}$ to $255 \mathrm{~nm} \cdot{ }^{26}$ Simultaneously to bending, the OCS molecule dissociates into S and CO. An advantage of studying the photodissociation dynamics of OCS lies in the fact that being an asymmetric triatomic molecule, symmetric and antisymmetric stretching vibrations can be uncoupled, and since the CO fragment is produced mainly in its electronic and vibrational ground state $\operatorname{CO}\left(X^{1} \Sigma^{+}, v=0\right),{ }^{27}{ }^{28}$ the stretching motion of the stiff CO bond can be considered a spectator during the dissociation. This significantly simplifies the understanding of the dissociation dynamics and allows for the use of a reduced dimensionality model. ${ }^{29}$ The photodissociation dynamics of OCS in its first absorption band has been extensively studied, both experimentally ${ }^{27-46}$ and theoretically. ${ }^{2947-51}$ Also the atmospheric relevance of OCS and its photodissociation as a sulphur source are of ongoing interest. 5253

For linear ground-state OCS dipole transitions to the excited ${ }^{1} \Sigma^{-}$and ${ }^{1} \Delta$ states are forbidden by symmetry. However, upon bending due to Renner-Teller interactions, degeneracies are lifted and the transitions become weakly allowed, with the transition strenghts increasing with increasing bending angle. ${ }^{54}$ In its first absorption band, OCS dissociates predominantly 
into

$$
\mathrm{OCS}+h \nu \longrightarrow \mathrm{CO}\left(X^{1} \Sigma^{+}\right)+\mathrm{S}\left({ }^{1} D_{2}\right)
$$

and

$$
\mathrm{OCS}+h \nu \longrightarrow \mathrm{CO}\left(X^{1} \Sigma^{+}\right)+\mathrm{S}\left({ }^{3} P_{J}\right), \quad J=0,1,2
$$

with a branching ratio of $95: 5$ at the center of the absorption band at $222 \mathrm{~nm} \cdot{ }^{29}$ In both channels, the $\mathrm{CO}$ fragment is mainly produced in its electronic and vibrational ground state. An upper bound for the population in the first excited vibrational state was given to be about $2 \% .{ }^{2728}$ The CO fragment produced in the major dissociation channel, together with a $\mathrm{S}\left({ }^{1} D_{2}\right)$ fragment, is highly rotationally excited, showing a bimodal rotational state distribution with two Gaussian-shaped underlying distributions. These distributions peak around $J=55$ and $J=67$ for photodissociation at $222 \mathrm{~nm}^{273132}$ and shift towards lower angular momenta for longer ultraviolet (UV)-excitation wavelength. ${ }^{27} 29$ The origin of the bimodal distribution was attributed to two different dissociation scenarios: The small- $J$-state component, measured at higher product kinetic energies, is generally assigned to direct dissociation from the $2{ }^{1} A^{\prime}$ $\left(1^{1} \Delta\right)$ and $1^{1} A^{\prime \prime}\left(1^{1} \Sigma^{-}\right)$states. The large- $J$-state component observed at low kinetic energies is due to the dissociation from the $2^{1} A^{\prime}\left(1^{1} \Delta\right)$ state alone, but involving non-adiabatic transitions to the $1^{1} A^{\prime}\left(X^{1} \Sigma^{+}\right)$ground state. ${ }^{29}$ For graphical representations of the energies of all electronic states involved we refer the reader to, e. g., Figures 1 in refs. 49, 55.

Although the dissociation process responsible for the formation of rotationally excited $\mathrm{CO}\left(X^{1} \Sigma^{+}\right)$and $\mathrm{S}\left({ }^{1} D_{2}\right)$ products is well understood and mainly attributed to prompt dissociation following the excitation of the $2{ }^{1} A^{\prime}\left(1^{1} \Delta\right)$ and $1{ }^{1} A^{\prime \prime}\left(1^{1} \Sigma^{-}\right)$states, the mechanisms responsible for the formation of $\mathrm{S}\left({ }^{3} P_{J}\right)$ fragments remains to be elucidated. Strong vibrational resonances were recently observed in the $2+1$ resonance-enhanced multi-photon ionisation (REMPI) spectra of the $\mathrm{S}\left({ }^{3} P_{J}\right)$ photoproducts recorded in a wavelength range from $212 \mathrm{~nm}$ to $260 \mathrm{~nm}$, which were assigned to vibrational progressions in the $\mathrm{C}-\mathrm{S}$ stretching following direct excitation to the $2^{1} A^{\prime \prime}\left(1^{1} \Delta\right)$ and triplet $2{ }^{3} A^{\prime \prime}\left(1^{3} \Sigma^{-}\right)$metastable states. ${ }^{55}$ Molecules in the $2^{3} A^{\prime \prime}\left(1^{3} \Sigma^{-}\right)$state can rapidly predissociate through non-adiabatic transitions to the repulsive $1^{3} A^{\prime \prime}$ state, leading to $\mathrm{S}\left({ }^{3} P_{J}\right)$ products. Alternatively, it has been predicted that the $2{ }^{1} A^{\prime \prime}\left(1^{1} \Delta\right)$ and $2{ }^{3} A^{\prime \prime}\left(1^{3} \Sigma^{-}\right)$states can predissociate through non-adiabatic coupling to 
the $1{ }^{1} A^{\prime \prime}\left(1^{1} \Sigma^{-}\right)$state or through spin-orbit coupling to the $2^{1} A^{\prime}\left(1^{1} \Delta\right)$ state, respectively, with subsequent intersystem crossing leading to the formation of $\mathrm{S}\left({ }^{3} P_{J}\right)$ fragments. ${ }^{55}$

So far, the experimental studies of the photodissociation of OCS were limited to frequencyresolved spectroscopies, i. e., with nanosecond lasers. This includes laser-induced fluorescence (LIF) spectroscopy ${ }^{273156}$ and REMPI. ${ }^{282932-3436-4657-59}$ These experiments, coupled to computational investigations, ${ }^{47-5060}$ have provided a wealth of detailed information on the photodissociation products, including their final state distributions. However, to date no time-resolved studies have been carried out to probe the transient electronic and nuclear structure of the molecule along the dissociation pathway. Starting with the present work, we try to bridge this gap. Here, the dissociation dynamics of the OCS molecule following UV excitation with a $237 \mathrm{~nm}$ femtosecond laser pulse is investigated. Reactants and products are probed by strong-field ionisation with a time-delayed $790 \mathrm{~nm}$ femtosecond laser pulse and the velocity distributions of $\mathrm{S}^{+}$and $\mathrm{CO}^{+}$fragment ions are imaged. Furthermore, quantum chemistry simulations taking into account the prompt dissociation following excitation of the $2^{1} A^{\prime}$ $\left(1^{1} \Delta\right)$ and $1^{1} A^{\prime \prime}\left(1^{1} \Sigma^{-}\right)$states to form $\mathrm{S}\left({ }^{1} D_{2}\right)$ products were coupled to a simple model for calculating strong-field-ionisation rates. Interestingly, this model was unable to reproduce the fast oscillations observed in the kinetic energy distribution of the $\mathrm{S}^{+}$fragments. Instead, our results provide strong indications that vibrational wavepackets are formed in the $2^{1} A^{\prime \prime}\left(1^{1} \Delta\right)$ and $2^{3} A^{\prime \prime}\left(1^{3} \Sigma^{-}\right)$states, which predissociate to form $\mathrm{S}\left({ }^{3} P_{J}\right)$. These experiments form the basis for ongoing investigations of the ultrafast photodissociation dynamics of OCS following UV excitation using structurally 232461 or electronic-state ${ }^{62}$ resolved laser-induced-electron diffraction and photoelectron imaging experiments.

\section{EXPERIMENTAL SETUP}

The experimental setup was described previously. ${ }^{61}$ Briefly, a cold molecular beam was formed by supersonic expansion of a 90 bar mixture of OCS (500 ppm) seeded in helium using a pulsed Even-Lavie valve ${ }^{63}$ operated at $250 \mathrm{~Hz}$. Thus, all molecules were in their electronic ground state, vibrationally cold, and their rotational temperature was characterised to be $T_{\text {rot }}=0.6 \mathrm{~K}$ at the interaction point of the molecules with the laser pulses. ${ }^{61}$

The output of a commercial Ti:Sapphire amplified laser system (KMLabs, Wyvern30), delivering $30 \mathrm{~mJ}, 38 \mathrm{fs}$ (FWHM) pulses at a central wavelength of $790 \mathrm{~nm}$ and a repetition 
rate of $1 \mathrm{kHz}$, was divided into three beams, whose time delays were controlled using motorised delay stages. Up to $20 \mathrm{~mJ}$ were used to pump an optical parametric amplifier (Light Conversion HE-TOPAS), generating ultrashort pulses at $1190 \mathrm{~nm}$ with a maximum output energy of $3.5 \mathrm{~mJ}$. A second path was used to generate UV light via second harmonic generation followed by frequency mixing with the second harmonic of the $1190 \mathrm{~nm}$ laser pulse. This yielded UV pulses with a wavelength of $237 \mathrm{~nm}$, a pulse duration of $70 \mathrm{fs}$ (FWHM), and a pulse energy of up to $15 \mu \mathrm{J}$. The third $790 \mathrm{~nm}$ (NIR) beam was used as the ionising probe pulse.

The UV-pump and NIR-probe pulses were temporally and spatially overlapped at the centre of a high-energy velocity-map-imaging spectrometer (VMI) with their polarisation parallel to the plane of the detector. $\mathrm{CO}^{+}$and $\mathrm{S}^{+}$ions were generated through strongfield ionisation of OCS or its dissociation products, accelerated into a $10 \mathrm{~cm}$ long field-free flight tube, and detected with the combination of a $77 \mathrm{~mm}$ diameter dual-microchannelplate/phosphor-screen assembly and a CCD camera. The projected two-dimensional (2D) ion-momentum distributions were inverted using an Abel-inversion procedure based on the BASEX algorithm ${ }^{64}$ in order to yield three-dimensional (3D) ion-momentum distributions.

Ion momentum distributions were recorded for $\mathrm{OCS}^{+}, \mathrm{S}^{+}$, and $\mathrm{CO}^{+}$fragments for a series of pump-probe time delays $\tau$ separated by $20 \mathrm{fs}$. Cross-correlation measurements of the parent ion were used to define the time overlap between the UV-pump and the NIR-probe pulse, i. e., $\tau=0$. The $\mathrm{S}^{+}$and $\mathrm{CO}^{+}$ion momentum distributions were recorded for various sets of UV $(4.5 \mu \mathrm{J}$ and $10 \mu \mathrm{J})$ and NIR $(300 \mu \mathrm{J}$ and $1.3 \mathrm{~mJ})$ pulse energies. All ion momentum distributions presented in this manuscript were averaged over, at least, 3500 laser shots.

\section{COMPUTATIONAL WAVEPACKET DYNAMICS}

The photodissociation of OCS in its first absorption band is known to mainly proceed by prompt dissociation following the excitation of the $2^{1} A^{\prime}\left(1^{1} \Delta\right)$ and $1^{1} A^{\prime \prime}\left(1^{1} \Sigma^{-}\right)$states. Therefore, in our calculations OCS molecules were vertically excited from the rovibronicground-state potential-energy surface (PES) to the $1^{1} A^{\prime \prime}\left(1^{1} \Sigma^{-}\right)$and the $2^{1} A^{\prime}\left(1^{1} \Delta\right)$ states, i. e., the ground-state density was projected onto these PESs. The PESs used are based on highlevel MRCI/aug-cc-pVQZ electronic structure calculations; ${ }^{47-50}$ see ref. 47, 49 for graphical representations. Following photoexcitation, the wavepackets were then propagated on these 


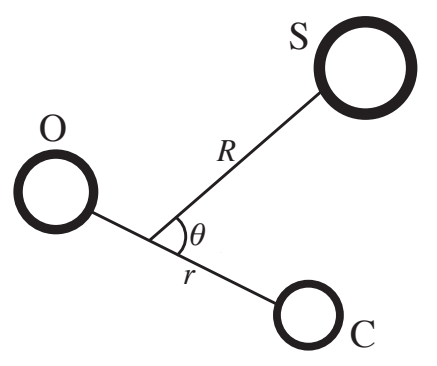

FIG. 1. Jacobi coordinates employed in the quantum dynamics simulations.

PESs using the multiconfigurational time-dependent Hartree approach (MCTDH). ${ }^{65} 66$ Jacobi coordinates as defined in Figure 1 were used throughout the quantum dynamics simulations, with $r$ denoting the $\mathrm{CO}$ bond length, $R$ the distance of the $\mathrm{S}$ atom to the centre of mass of the carbon monoxide fragment, and $\theta$ the angle between $r$ and $R .{ }^{6768}$ The wavepackets were propagated over 400 fs, using adaptive step sizes, with the photoexcitation taking place at $t=0$.

The ansatz for a set of $\mathrm{MCTDH}^{65}$ wave functions is given by (1) in ref. 66, with the so-called single-particle functions (SPF) as time-dependent basis functions. The SPFs are expanded in a time-independed basis, see ref. 66, (2). The degrees of freedom in our calculations employed here are the coordinates $R, r$, and $\theta$, as described above.

Employing fast-Fourier-transform (FFT) or discrete-variable-representation (DVR) schemes for distances and angles, respectively, the SPFs were expanded in a time-independent basis. The correlation DVR (CDVR $)^{69}$ scheme was used to obtain matrix elements for general potential energy surfaces. Throughout this work, the revised constant mean-field scheme $(\mathrm{CMF} 2)^{70}$ was used to efficiently propagate the MCTDH wavepackets. The converged basis set sizes are given in Table I. Please note that slightly smaller grids are employed for calculating the OCS ground state.

\begin{tabular}{ccccc}
\hline \hline coordinate & expansion type & $n$ & $N$ & range \\
\hline$R$ & FFT & 16 & 2048 & $2.8-72.0$ a.u. \\
$r$ & FFT & 7 & 48 & $1.5-3.0$ a.u. \\
$\theta$ & Legendre-DVR & 22 & 128 & $0-\pi$ \\
\hline \hline
\end{tabular}

TABLE I. Expansion type, number of grid points $N$, and number of single-particle functions $n$ used in the presented quantum dynamics simulations. 


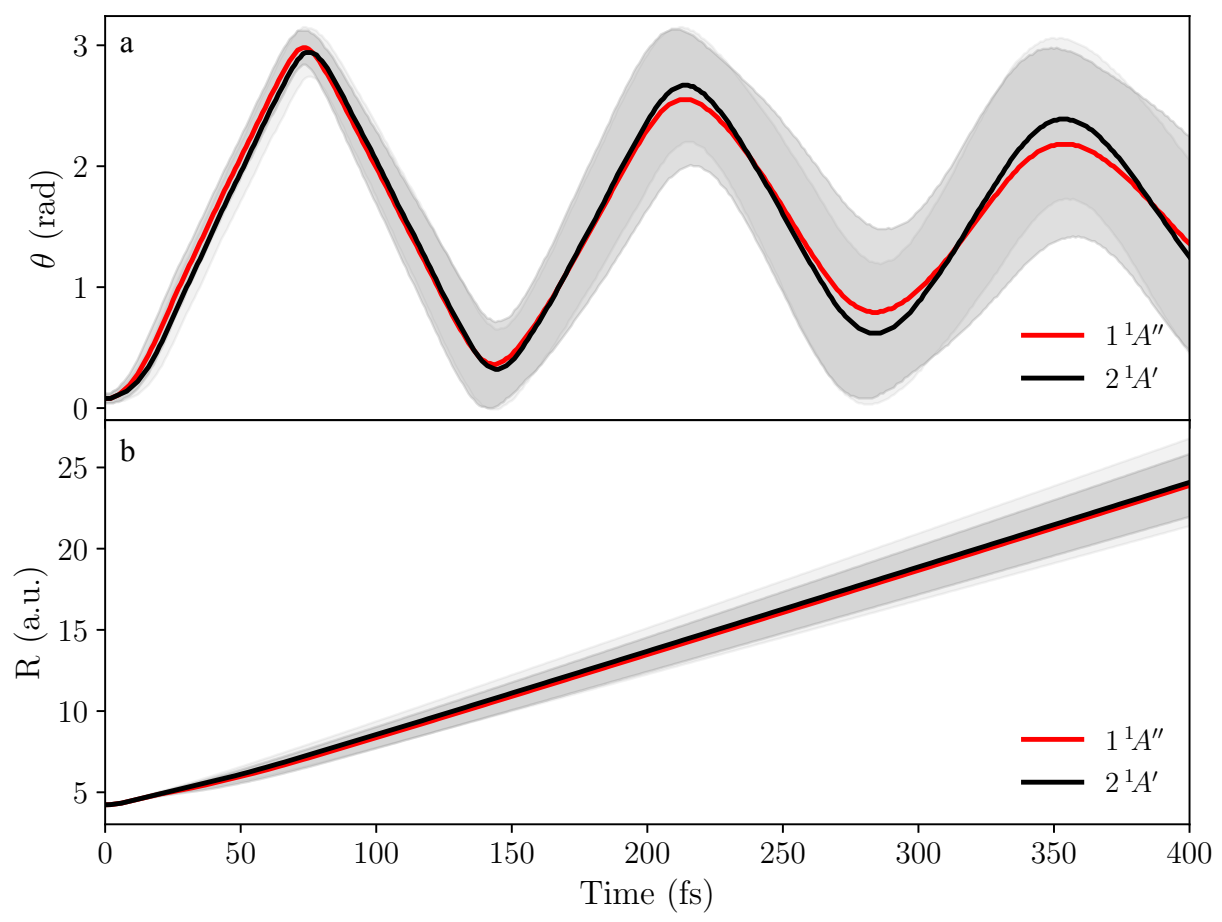

FIG. 2. Time-dependent expectation values of (a) $\theta$ and (b) $R$ for the computed wavepackets on the $1^{1} A^{\prime \prime}$ and $2{ }^{1} A^{\prime}$ potential-energy surfaces; see Figure 1 and Section III for details. Standard deviations of the calculated probability densities are depicted as grey areas to show the spread of the wavepackets.

Movies of the wavepacket dynamics along the reaction coordinates obtained from our model in the $2^{1} A^{\prime}\left(1^{1} \Delta\right)$ and $1^{1} A^{\prime \prime}\left(1^{1} \Sigma^{-}\right)$excited states are provided in the supplementary material. Figure 2 shows the calculated time-dependent expectation values of $\theta$ and $R$ for the calculated wavepacket dynamics on the $1^{1} A^{\prime \prime}\left(1^{1} \Sigma^{-}\right)$and $2^{1} A^{\prime}\left(1^{1} \Delta\right)$ potential energy surfaces, which are very similar. The temporal evolution of the wavepackets in these electronic states exhibits a monotonic increase of $R$, Figure $2 \mathrm{~b}$. For $t=100 \mathrm{fs}, R$ is already twice the equilibrium distance and from there on increases nearly linearly, implying that the chemical bond is effectively being broken already at this time.

The time-dependent expectation values of $\theta$, see Figure 2 a, shows pronounced oscillations with a period of $T=140$ fs. These oscillations can be assigned to the rotational motion of the CO product resulting from the bending of the molecule following UV-excitation and dissociation of OCS. The observed rotational period corresponds to the classical rotation period of a state with rotational quantum number $J \approx 61$, which fits previously observed ${ }^{29}$ 
terminal CO rotational-state distributions very well.

\section{RESULTS AND DISCUSSION}

Figure 3 shows slices through the 3D ion-momentum distributions of $(\mathrm{a}-\mathrm{c})$ the $\mathrm{S}^{+}$and $(\mathrm{d}-\mathrm{f}) \mathrm{CO}^{+}$fragments recorded upon photoionisation of UV-excited OCS as well as (g, h) the corresponding angle-integrated kinetic energy spectra. In these measurements the pulse energies of the UV-excitation pulses $(237 \mathrm{~nm})$ and the NIR ionisation pulses were set to $4.5 \mu \mathrm{J}$ and $300 \mu \mathrm{J}$, respectively. The $\mathrm{S}^{+}$momentum distribution is composed of two main channels that peak at kinetic energies of $0.22 \mathrm{eV}$ and $2.53 \mathrm{eV}$. The $0.22 \mathrm{eV}$ channel is assigned to ionisation of OCS into low-energy excited states of the molecular $\mathrm{OCS}^{+}$cation, which are known to dissociate to form a singly charged $\mathrm{S}$ atom and a neutral $\mathrm{CO}$ fragment in its electronic ground state. ${ }^{71}$ The $2.53 \mathrm{eV}$ channel corresponds to Coulomb explosion following double ionisation of the molecules by the NIR pulses leading to two singly charged $\mathrm{S}^{+}$and $\mathrm{CO}^{+}$fragments. In addition, a weaker and broader tail around $5 \mathrm{eV}$ is observed, which is due to Coulomb explosion with a doubly, or higher, charged $\mathrm{CO}^{n+}(n \geq 2)$ counterion.

Similarly, in the $\mathrm{CO}^{+}$angle-integrated kinetic energy spectrum, Figure $3 \mathrm{~h}$, three channels are observed at kinetic energies of $0.33 \mathrm{eV}, 2.84 \mathrm{eV}$, and $5.26 \mathrm{eV}$. Complementary to $\mathrm{S}^{+}$, the lowest energy channel can be attributed to the ionisation of the OCS molecule followed by dissociation leading to a singly charged $\mathrm{CO}^{+}$fragment and a neutral $\mathrm{S}$ atom, ${ }^{71}$ whereas the two other channels can be assigned to Coulomb explosion of the molecules by the NIR laser pulses with $\mathrm{S}^{+}$and $\mathrm{S}^{2+}$ as counterions. Based on the intensities of the lowest kinetic-energy peaks in the spectra in Figure $3 \mathrm{~g}, \mathrm{~h}$, the dissociation of $\mathrm{OCS}^{+}$predominantly produces $\mathrm{S}^{+}$ ions, in agreement with previous single photon ionisation experiments. ${ }^{71}$

When the UV pulse precedes the NIR pulse a strong enhancement of the ion signal is observed for both, $\mathrm{S}^{+}$and $\mathrm{CO}^{+}$, fragments in the dissociative-ionisation channel and the first Coulomb explosion channel, i. e., the channels with one or two overall charges in the system. This can be rationalised by the fact that the UV-excited OCS has a much smaller ionisation energy $\left(E_{i}\right)$ and can be ionised with significantly weaker fields/less NIR

photons. The large increase of the signal observed in the dissociative-ionisation channel of both fragments can a priori be assigned to UV-excitation to the $2^{1} A^{\prime}\left(1^{1} \Delta\right)$ and $1{ }^{1} A^{\prime \prime}\left(1^{1} \Sigma^{-}\right)$ states, which dissociate quickly to form ground state $\operatorname{CO}\left(X^{1} \Sigma^{+}\right)$and electronically excited 
$\mathrm{S}\left({ }^{1} D_{2}\right)$ fragments, ${ }^{2729}$ followed by ionisation of one of the fragments by the NIR laser pulses. Considering that the ionisation energy of $\mathrm{S}\left({ }^{1} D_{2}\right), E_{i} \approx 9.2 \mathrm{eV}$, is much lower than the one of $\mathrm{CO}\left(X^{1} \Sigma^{+}\right), E_{i} \approx 14 \mathrm{eV}$, the NIR pulse is expected to preferentially ionise the $\mathrm{S}$ atom.
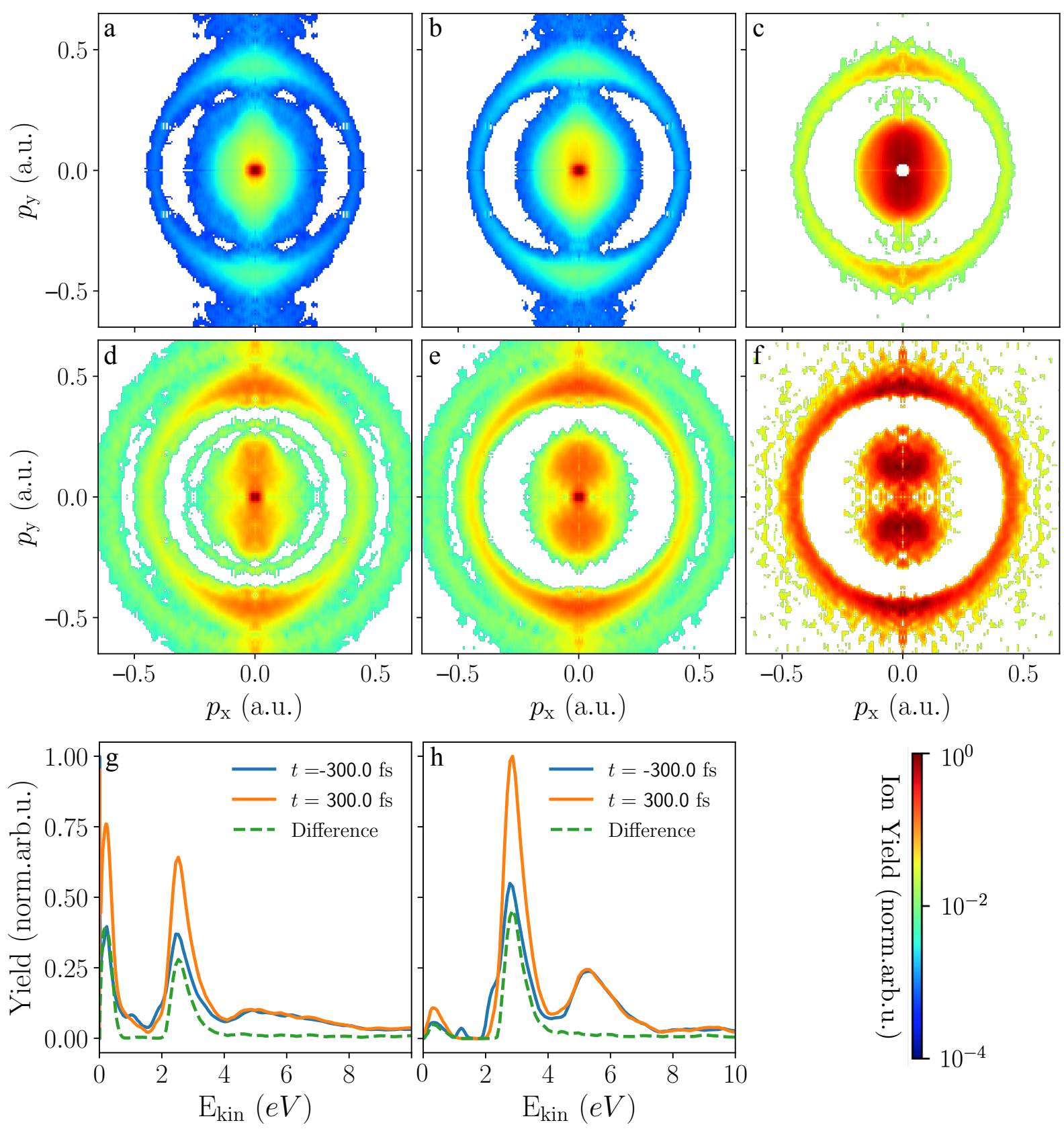

FIG. 3. Slices through 3D Abel-inverted ion-momentum distributions of $\mathrm{S}^{+}$and $\mathrm{CO}^{+}$fragments and corresponding angle-resolved kinetic energy spectra. $(\mathrm{a}-\mathrm{c}, \mathrm{g}) \mathrm{S}^{+}$and $(\mathrm{d}-\mathrm{f}, \mathrm{h}) \mathrm{CO}^{+}$ion momentum distributions recorded at a UV-NIR time delay of (a,d) $\tau=-300$ fs and (b, e) $\tau=+300$ fs, $(\mathrm{c}, \mathrm{f})$ their differences, and $(\mathrm{g}, \mathrm{h})$ the corresponding angle-integrated kinetic-energy distributions. 
This trend is clearly observed experimentally as the dissociative-ionisation signal observed at positive time delays is larger in the $\mathrm{S}^{+}$channel than in the $\mathrm{CO}^{+}$channel.

In principle, one might expect that the large increase of the signal in the first Coulomb explosion channel similarly originates from the ionisation of two co-fragments formed following UV excitation and dissociation of the neutral molecules. However, in the case of Coulomb explosion from dissociating OCS molecules, we would expect the kinetic energy of the $\mathrm{S}^{+}$ and $\mathrm{CO}^{+}$fragments to decrease with increasing time delay, as the Coulomb repulsion energy decreases as $\sim 1 / R$ when the internuclear distance $R$ increases ${ }^{72}$ which is not observed here, see also Figure 4. Instead, the large increase of the ionisation yield in the first Coulomb explosion channel suggests that a large number of OCS molecules remain bound after UV-excitation.

The ion yield observed in the highest-kinetic energy channels, due to Coulomb explosion of an OCS system with overall three (or more) charges, is identical at UV-NIR time delays of $\tau=-300$ fs and $\tau=+300$ fs. This is seen in the differences of the momentum maps in Figure $3 \mathrm{c}, \mathrm{f}$, where the corresponding outermost rings for these Coulomb explosion channels are not present, and the corresponding kinetic energy spectra in Figure $3 \mathrm{~g}, \mathrm{~h}$, where the spectra are identical at these high kinetic energies. This could be due to the fact that these high-kinetic energy channels require the removal of at least three electrons, including electrons from deeper orbitals than the HOMO, and, therefore, are little affected by the initial UV-excitation of an electron from the HOMO orbital into unoccupied orbitals of the neutral OCS.

In Figure 4 the delay-dependent kinetic energy spectra for $\mathrm{S}^{+}$and $\mathrm{CO}^{+}$fragments recorded in the delay interval $\tau=-300 \ldots 500$ fs are shown. In the following discussion we focus on the two lower-kinetic-energy channels of both ions, i.e., below $3 \mathrm{eV}$, as the higher-energy channels only exhibit effects when the two pulses overlap in time.

A large enhancement of the ion signal is observed for both ionic fragments when the two pulses overlap in time. This increase around $\tau=0$ is attributed to the increased efficiency of two-color multiphoton ionisation when the two laser fields are present simultaneously. At positive time delays, the signal levels for the two lowest ion kinetic energy channels observed in both fragments remain nearly constant and are approximately two times larger than for negative delays.

The large increase of the dissociative-ionisation signal, i. e., in the lowest-kinetic-energy 


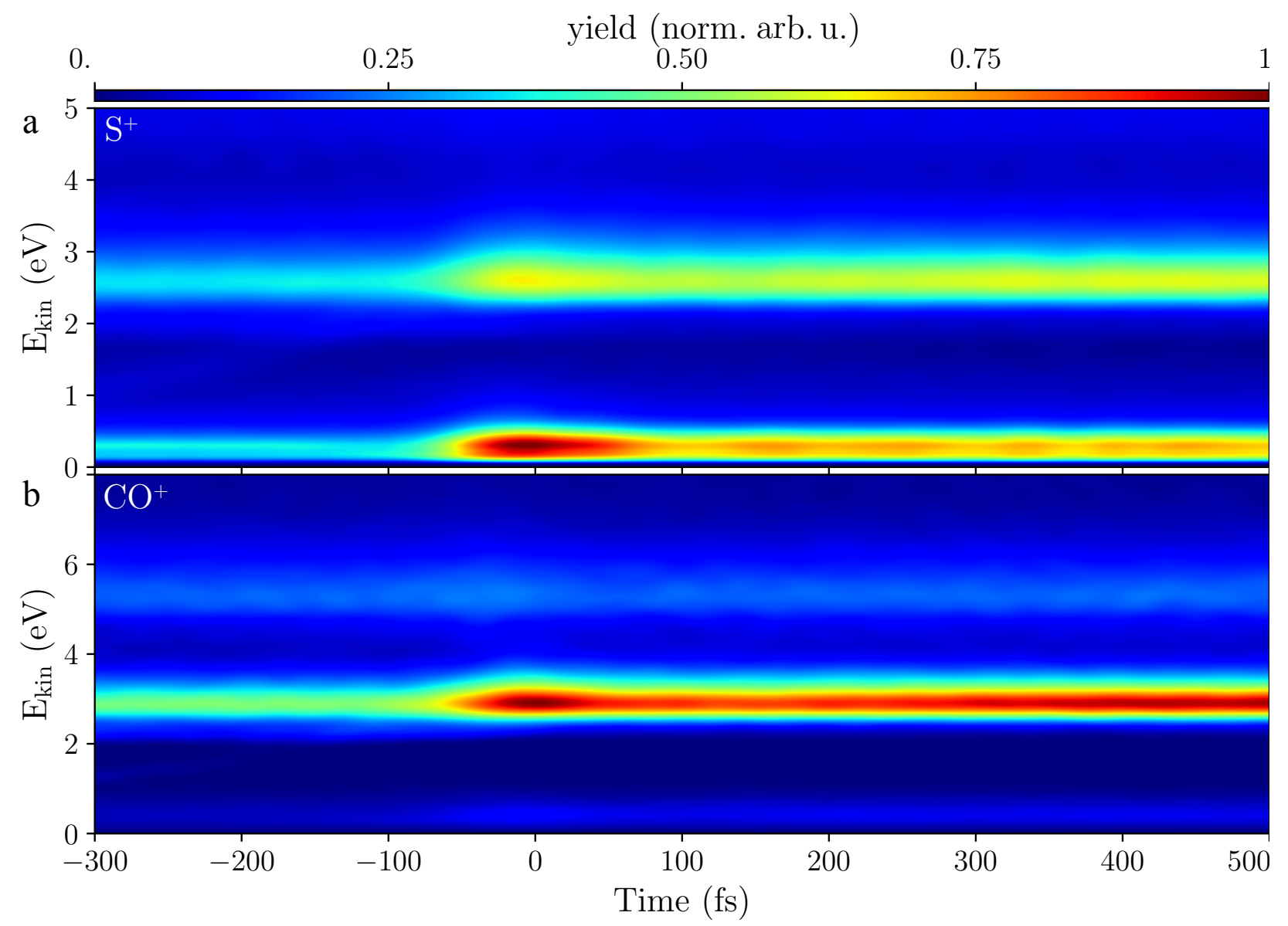

FIG. 4. Time-dependent angle-integrated kinetic energy spectra of (a) $\mathrm{S}^{+}$and (b) $\mathrm{CO}^{+}$fragments in the time interval $\tau=-300 \ldots 500$ fs. A positive delay means the UV pulse precedes the NIR pulse.

channels at positive time delays, with the major contribution in $\mathrm{S}^{+}$, provides information on the dissociation dynamics in the UV-excited OCS molecule. As mentioned above, the main contribution is from excitation to the $2^{1} A^{\prime}\left(1^{1} \Delta\right)$ and $1^{1} A^{\prime \prime}\left(1^{1} \Sigma^{-}\right)$states, which quickly dissociate into S and CO. This was confirmed by the results of our wavepacket calculations, which showed that at $t=100$ fs the bond is effectively broken, see Section III. Thus, the NIR pulse will ionise the produced neutral $\mathrm{S}$ or $\mathrm{CO}$ fragments with a preference for the $\mathrm{S}$ atom due to its much lower $E_{i}$. We note that dissociation dynamics occuring within the first 100 fs following UV-excitation cannot be unravelled in our measurements due to the relatively long pulse duration ( $\sim 70 \mathrm{fs})$ of the $237 \mathrm{~nm}$ pulse.

The time dependence of the Coulomb explosion channel resulting in $\mathrm{S}^{+}$and $\mathrm{CO}^{+}$with kinetic energies of $2.53 \mathrm{eV}$ and $2.84 \mathrm{eV}$, respectively, cannot be explained by NIR ionisation 
of neutral fragments that result from prompt dissociation of the UV-excited OCS molecules: such a process would have given rise to a kinetic energy spectra where the kinetic energy release decreases with pump-probe time delay, which is not the case in the experimental data, see Figure 4. Instead, we tentatively assign the large observed increase of the Coulomb explosion channel for $\tau>0$ to the UV-excitation of bound electronically excited states, e. g., the $2{ }^{1} A^{\prime \prime}\left(1^{1} \Delta\right), 2^{3} A^{\prime}\left(1^{3} \Delta\right)$, and $2^{3} A^{\prime \prime}\left(1^{3} \Sigma^{-}\right)$triplet states, that are accessible at a photon energy of $237 \mathrm{~nm}$. Due to their lower $E_{i}$, these excited OCS molecules are readily multiply ionised by the probe IR pulse, enhancing their contribution in the kinetic-energy spectra. A similarly increased probability for dissociative single ionisation could also partly be responsible for the increased $\mathrm{S}^{+}$and $\mathrm{CO}^{+}$signals observed in the lowest kinetic-energy channels.

In addition to the large increase of the $\mathrm{S}^{+}$and $\mathrm{CO}^{+}$ionisation yields, weak but pronounced oscillations are observed at positive time delays in the dissociative-ionisation channels of both $\mathrm{S}^{+}$and $\mathrm{CO}^{+}$. Integrating the ionisation yields around the lowest kinetic energy channels observed in the $\mathrm{S}^{+}$and $\mathrm{CO}^{+}$fragments, i. e., at kinetic energies of $0.22 \mathrm{eV}$ and $0.33 \mathrm{eV}$ yields the time-dependent ion yields in Figure 5. The oscillations exhibit a period of $T_{\mathrm{S}^{+}} \approx 100 \mathrm{fs}$ for $\mathrm{S}^{+}$. For the $\mathrm{CO}^{+}$channel the oscillation period seems to be longer, but no well-defined value could be extracted as no full period is observed within the time interval that the $\mathrm{CO}^{+}$ fragments were recorded. The observed oscillations are independent of the UV and NIR pulse energies used, which suggests that the observed dynamics is not due to two-photon excitations to higher excited states of neutral OCS.

The dissociation dynamics of OCS in the $1^{1} A^{\prime \prime}$ and $2^{1} A^{\prime}$ potential energy surfaces is known to involve large bending motion of the molecule, which results in CO products with high rotational excitation. ${ }^{27} 29$ This rotational excitation was also clearly observed in our wavepacket calculations, i. e., the time-dependent expectation value of $\theta$ shown in Figure 2 a. Building upon these computational results we attempt to explain the oscillations observed in ion yields, esp. the time-dependent $\mathrm{S}^{+}$yields using an intuitive physical picture: One would expect that during dissociation the dipole moment of the rotating $\mathrm{CO}$ influences the instantaneous ionisation rate of the nearby S atom: the direction of the rotating dipole with respect to the $R$ coordinate shifts the ionisation potential of the sulfur atom, and thus its ionisation rate. To assess whether this effect is responsible for the observed oscillations, we used a simple ADK-tunnel-ionisation model ${ }^{73}$ to simulate the expected modulation of the 


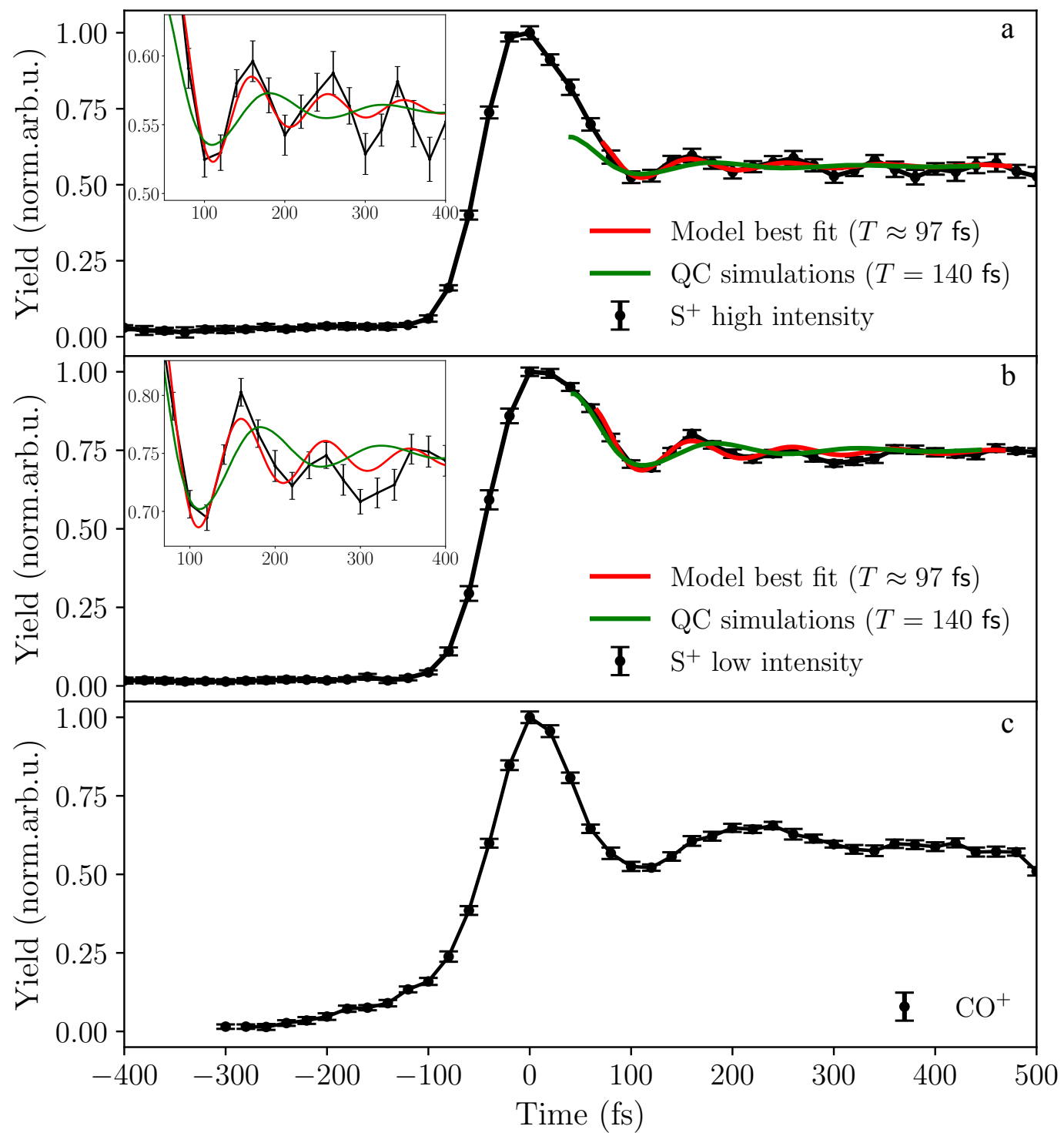

FIG. 5. Time-dependent $\mathrm{S}^{+}$and $\mathrm{CO}^{+}$yields for the delay range $\tau=-400 \ldots 500$ fs. (a, b) $\mathrm{S}^{+}$ions with a kinetic energy of $\sim 0.22 \mathrm{eV}$ for UV and NIR pulse energies of (a) $10 \mu \mathrm{J}$ and $1.3 \mathrm{~mJ}$ and (b) $4.5 \mu \mathrm{J}$ and $300 \mu \mathrm{J}$, respectively. The (black) experimental $\mathrm{S}^{+}$yields are compared to (red and green) simulations considering a modified ADK ionisation rate for the sulfur atom due to the rotational motion of the $\mathrm{CO}$ fragment, see the text for details. The insets show a magnified view on the oscillations for better visibility. (c) $\mathrm{CO}^{+}$-ions with a kinetic energy of $\sim 0.38 \mathrm{eV}$ for $\mathrm{UV}$ and NIR pulse energies of $10 \mu \mathrm{J}$ and $1.3 \mathrm{~mJ}$, respectively.

S-atom ionisation rate as a function of time under the influence of the rotating CO dipole. For time delays $\tau>100 \mathrm{fs}$, approximately the time-scale for the onset of the observed oscillations, $R \gtrsim 9$ a. u. and thus $\mathrm{S}$ can be considered to be a quasi-free atom. The ionisation signal can 
then be well approximated by the strong-field-ionisation rate of $\mathrm{S}$ in the field of the $\mathrm{CO}$ dipole moment. We calculated the electric potential of the CO dipole moment at the position of the S atom as $V(t)=\mu \cos \theta(t) / R(t)$ with the angle $\theta(t)$ of the rotating CO fragment at time $t$ and the dipole moment of $\mathrm{CO} \mu=0.122 \mathrm{D}$. We note that this expression only holds for $R \gg r$, see Figure 1 for the coordinates. However, we also benchmarked the results against further multipole expansions and even explicit partial charges on $\mathrm{C}$ and $\mathrm{O}$, which did not make any difference. For $R \approx 10 \mathrm{a}$. u. the change of $E_{i}$ is on the order of $0.1 \mathrm{eV}$. The time-dependent ionisation rate was computed once using the time-dependent expectation values for $\theta(t)$ from our wavepacket simulations, Figure $2 \mathrm{a}$, and once by fitting the time-period for the rotational motion of $\mathrm{CO}$ to the experimental curves by employing a least-squares minimisation. In both cases, the time-dependent change of the CO-S internuclear distance $R(t)$ was taken directly from the results of our wavepacket simulations on the $1{ }^{1} A^{\prime \prime}$ and $2{ }^{1} A^{\prime}$ potential energy surfaces, see Figure $2 \mathrm{~b}$.

The results of this model are shown in Figure $5 \mathrm{a}, \mathrm{b}$, in comparison with the experimentally measured time-dependent $\mathrm{S}^{+}$dissociative ionisation yields recorded with (a) low and (b) high UV and NIR pulse energies, respectively. The rotational period of $T=140 \mathrm{fs}$, obtained from the quantum chemistry simulations, directly translates into a $T_{\mathrm{S}^{+}}=140$ fs period in the ionisation signal and, therefore, does not match the measured oscillations. When used as a fitting parameter, a rotational period of $T=97_{-8}^{+14}$ fs is retrieved for the two intensities. This fast rotational period would correspond to the population of rotationally excited CO fragments with $J>80$, which clearly disagrees with earlier REMPI spectra of the S photoproducts. ${ }^{28} 29313244-46$ Therefore, we conclude that the observed oscillations are not caused by the electrostatic interaction of the highly rotationally excited CO fragment with the neutral $\mathrm{S}$ atom along the dissociation pathway.

Instead, the fast oscillations observed in the $\mathrm{S}^{+}$products of the UV-induced dissociation can be explained by the population of vibrational wavepackets in one or more electronically excited states of OCS, for which bound vibrational states are accessible at $237 \mathrm{~nm}$. REMPI spectra of the $\mathrm{S}$ photoproducts showed a broad, unstructured spectrum in the major $\mathrm{S}\left({ }^{1} D_{2}\right)$ channel. ${ }^{55}$ This suggests that the absorption spectrum and correspondingly the observed products are dominated by excitation to the repulsive $2^{1} A^{\prime}\left(1^{1} \Delta\right)$ and $1^{1} A^{\prime \prime}\left(1^{1} \Sigma^{-}\right)$states, which lead to prompt dissociation, see also Figure 2. The repulsive nature of these two states along the dissociation coordinate was confirmed by earlier calculations ${ }^{47}$ However, these 
calculations also indicated that these states have shallow local minima supporting bound vibrational states around bending angles of $\theta \approx 40^{\circ}$. The profound vibrational structure observed in the low-energy tail of the absorption spectrum of OCS, e.g., above $\sim 270 \mathrm{~nm}$, was assigned to these states. ${ }^{55}$ Although in our experiment we excite the OCS molecules to much higher energies, we cannot fully rule out the formation of vibrational wavepackets in these $2{ }^{1} A^{\prime}\left(1^{1} \Delta\right)$ and $1^{1} A^{\prime \prime}\left(1^{1} \Sigma^{-}\right)$states, which would predissociate through non-adiabatic coupling to the $1^{1} A^{\prime}$ ground state.

In addition, a strong vibrational progression superimposed on a broad continuum was recently observed in the REMPI spectrum of the minor $\mathrm{S}\left({ }^{3} P_{J}\right)$ products following UVexcitation of OCS in its first absorption band..$^{55}$ The diffuse vibrational structure was assigned to direct excitation to the bound $2^{1} A^{\prime \prime}\left(1^{1} \Delta\right)$ and $2^{3} A^{\prime \prime}\left(1^{3} \Sigma^{-}\right)$metastable states. The $2^{3} A^{\prime \prime}\left(1^{3} \Sigma^{-}\right)$triplet state can predissociate through spin-orbit coupling to the dissociative $2{ }^{1} A^{\prime}\left(1^{1} \Delta\right)$ state, with subsequent intersystem crossing to form $\mathrm{S}\left({ }^{3} P_{J}\right)$ products. Similarly, the $2{ }^{1} A^{\prime \prime}\left(1^{1} \Delta\right)$ state can predissociate through rovibronic coupling to the dissociative $1{ }^{1} A^{\prime \prime}$ $\left(1^{1} \Sigma^{-}\right)$, with subsequent intersystem crossing to the $2{ }^{3} A^{\prime \prime}\left(1^{3} \Sigma^{-}\right)$to form $\mathrm{S}\left({ }^{3} P_{J}\right)$ products at larger $R$. Interestingly, a weak absorption feature was observed at a UV wavelength of $236.12 \mathrm{~nm}$, i.e., in the central part of our UV pump-laser wavelength, in the REMPI spectrum of the minor $\mathrm{S}\left({ }^{3} P_{J}\right)$ product. ${ }^{55}$ This band was assigned to the $\mathrm{C}-\mathrm{S}$ stretching vibration $\nu_{1}$ of the $2^{1} A^{\prime \prime}\left(1^{1} \Delta\right)$ state. It strongly suggests that the fast oscillations of the $\mathrm{S}^{+}$and $\mathrm{CO}^{+}$products in our time-resolved measurements are due to vibrational wavepacket dynamics in the $2{ }^{1} A^{\prime \prime}\left(1^{1} \Delta\right)$ states, which then predissociate to form $S\left({ }^{3} P_{J}\right)$ products. This UV excitation to bound states is also corroborated by the UV-induced enhancements in the Coulomb-explosion channels discussed above.

Further experiments and theoretical investigations are clearly needed to draw definite conclusions on the underlying physical process leading to the observed oscillations in the $\mathrm{S}^{+}$ signal. We are currently experimentally investigating the OCS reaction system using laserinduced electron diffraction in the molecular frame. ${ }^{23-2561}$ Imaging the dynamics observed here with our previously achieved 5 pm spatial ${ }^{23}$ and sub-100 fs temporal resolution would provide a very direct structural view on the ongoing dynamics. Alternatively, $\mathrm{MeV}$ ultrafast electron diffraction with sufficient time resolution is also within reach ${ }^{22}$ and could provide significant information on this structurally simple model system.

Complementary theoretical studies would undoubtedly be needed as well. In the vicinity 
of the Franck-Condon point, at least eight electronic states are known to be involved in the dissociation dynamics of OCS (four singlets and four triplets). ${ }^{49}$ Additional electronic states correlate to the two relevant dissociation limits, $\mathrm{S}\left({ }^{1} D_{2}\right)$ and $\mathrm{S}\left({ }^{3} P_{J}\right)$, which may become involved as the system dissociates. Due to the presence of the second-main-row S atom, the spin-orbit and non-adiabatic couplings are expected to be comparable, leading to both, direct excitation of the triplet manifold ${ }^{49}$ and rapid inter-system crossing and re-crossing. In the related, simpler case of bound dynamics in $\mathrm{SO}_{2}$, the non-adiabatic and spin-orbit coupling were found to be intertwined ${ }^{74}$ and both had to be treated to reach an agreement with experiment. ${ }^{75}$ Furthermore, the energy surfaces, and especially the transition dipoles in OCS are known to be highly sensitive to the treatment of electron correlation, ${ }^{50}$ potentially requiring correlation treatment at the coupled-cluster-triples level to achieve a satisfactory agreement with the experiment. ${ }^{60}$ Finally, at the high rotational excitation levels observed for the CO dissociation products, the reduced-dimensionality model may become insufficient. ${ }^{76}$ Although challenging, the multi-surface, full-dimensional description of the dissociation dynamics of photoexcited OCS is within reach of the ab initio electronic structure and MCTDH simulations.

\section{CONCLUSIONS AND OUTLOOK}

The ultrafast photodissociation dynamics of OCS following UV-photoexcitation at $\lambda=237 \mathrm{~nm}$ was investigated by time-resolved ion-momentum spectroscopy using a strong, time-delayed, $790 \mathrm{~nm}$ laser pulse. We observed a strong enhancement of the ion yields in the $\mathrm{S}^{+}$and $\mathrm{CO}^{+}$fragments due to UV excitation. This is consistent with the excitation of the molecules to the repulsive $2{ }^{1} A^{\prime}\left(1^{1} \Delta\right)$ and $1^{1} A^{\prime \prime}\left(1^{1} \Sigma^{-}\right)$states followed by prompt dissociation leading to $\mathrm{S}\left({ }^{1} D\right)$ and rotationally excited $\mathrm{CO}\left(X^{1} \Sigma^{+}\right)$photofragments. A pronounced increase of the Coulomb explosion ionisation rate following UV excitation of the molecules was also observed. This was assigned to the excitation of molecules in bound electronically excited states, most likely the $2^{1} A^{\prime \prime}\left(1^{1} \Delta\right)$ and $2^{3} A^{\prime \prime}\left(1^{3} \Sigma^{-}\right)$states. From a time delay of 100 fs onward fast oscillations of the $\mathrm{S}^{+}$and $\mathrm{CO}^{+}$yields were observed on top of a large time-independent signal, with a well-defined period of 100 fs in the $\mathrm{S}^{+}$signals.

Furthermore, we performed wavepacket dynamics simulations on the $2^{1} A^{\prime}\left(1^{1} \Delta\right)$ and $1^{1} A^{\prime \prime}$ $\left(1^{1} \Sigma^{-}\right)$states, which showed direct dissociation with fast rotation of the CO products with 
a 140 fs period. Using a simple electrostatic interaction and strong-field-ionisation model, we could rule out that this $\mathrm{CO}$ rotation produces the observed oscillations. Instead, these oscillations might reflect vibrational wavepacket dynamics in the electronically excited bound $2{ }^{1} A^{\prime \prime}\left(1^{1} \Delta\right)$ and $2^{3} A^{\prime \prime}\left(1^{3} \Sigma^{-}\right)$metastable states, which can predissociate by spin-orbit or rovibronic couplings to the minor dissociation channel leading to ground state $\mathrm{S}\left({ }^{3} P_{J}\right)$. This is in agreement with pronounced vibrational progressions in REMPI spectra of the $\mathrm{S}\left({ }^{3} P_{J}\right)$ products in the same excitation-energy range. ${ }^{55}$ Clearly, additional wavepacket dynamics simulations including the different electronic states that are known to be involved in the dissociation dynamics of OCS, as well as their couplings, are needed to understand the mechanism responsible for the observed fast oscillations of the $\mathrm{S}^{+}$dissociative ion yield.

This first time-resolved investigation of the photodissosiation dynamics of OCS clearly demonstrates the need for further advanced experimental and theoretical studies to elucidate the underlying physical processes responsible for the fast oscillations observed experimentally. Time-resolved electron-ion coincidence spectroscopy with extreme-ultraviolet laser pulses as a probe ${ }^{77}$ could provide further information on the investigated ultrafast dissociation process. Furthermore, experimental studies providing atomically-resolved images of the structural changes during these UV-induced dynamics could be very valuable. As a first step toward this goal, we have recently retrieved the structure of OCS in its equilibrium geometry using laser-induced electron diffraction ${ }^{23}$ and time-resolved LIED studies of the OCS photodissociation dynamics are underway to provide a first molecular movie of this benchmark system.

\section{ACKNOWLEDGEMENTS}

We thank Johan Schmidt for providing us with the numerical data of the potential energy surfaces used in our wavepacket calculations.

This work has been supported by the Deutsche Forschungsgemeinschaft (DFG) through the priority program "Quantum Dynamics in Tailored Intense Fields" (QUTIF, SPP1840, AR 4577/4, KU 1527/3) and by the Cluster of Excellence "Advanced Imaging of Matter" (AIM, EXC 2056, ID 390715994) of the Deutsche Forschungsgemeinschaft. 


\section{AUTHOR CONTRIBUTIONS}

J.K. and A.R. conceived and planned the experiment, E.T.K., S.Y., and A.R. performed the experiment, E.T.K. and S.Y. analysed the data, R.W. performed the wavepacket calculations, E.T.K., S.P., R.W., J.K., and A.R. interpreted the findings, E.T.K., J.K., and A.R. drafted the manuscript, and all authors discussed the results and contributed to the final manuscript.

\section{CONFLICTS OF INTEREST}

The authors declare no conflicts of interest.

\section{REFERENCES}

${ }^{1}$ A. H. Zewail, "Femtochemistry: Atomic-Scale Dynamics of the Chemical Bond," J. Phys. Chem. A 104, 5660-5694 (2000).

${ }^{2}$ L. Young, K. Ueda, M. Gühr, P. H. Bucksbaum, M. Simon, S. Mukamel, N. Rohringer, K. C. Prince, C. Masciovecchio, M. Meyer, A. Rudenko, D. Rolles, C. Bostedt, M. Fuchs, D. A. Reis, R. Santra, H. Kapteyn, M. Murnane, H. Ibrahim, F. Légaré, M. J. J. Vrakking, M. Isinger, D. Kroon, M. Gisselbrecht, A. L'Huillier, H. J. Wörner, and S. R. Leone, "Roadmap of ultrafast x-ray atomic and molecular physics," J. Phys. B 51, 032003 (2018) ${ }^{3}$ A. Barty, J. Küpper, and H. N. Chapman, "Molecular imaging using x-ray free-electron lasers," Annu. Rev. Phys. Chem. 64, 415-435 (2013).

${ }^{4}$ K. Pande, C. D. M. Hutchison, G. Groenhof, A. Aquila, J. S. Robinson, J. Tenboer, S. Basu, S. Boutet, D. P. DePonte, M. Liang, T. A. White, N. A. Zatsepin, O. Yefanov, D. Morozov, D. Oberthuer, C. Gati, G. Subramanian, D. James, Y. Zhao, J. Koralek, J. Brayshaw, C. Kupitz, C. Conrad, S. Roy-Chowdhury, J. D. Coe, M. Metz, P. L. Xavier, T. D. Grant, J. E. Koglin, G. Ketawala, R. Fromme, V. Šrajer, R. Henning, J. C. H. Spence, A. Ourmazd, P. Schwander, U. Weierstall, M. Frank, P. Fromme, A. Barty, H. N. Chapman, K. Moffat, J. J. van Thor, and M. Schmidt, "Femtosecond structural dynamics drives the trans/cis isomerization in photoactive yellow protein," Science 352, 725-729 (2016)

${ }^{5}$ J. Küpper, S. Stern, L. Holmegaard, F. Filsinger, A. Rouzée, A. Rudenko, P. Johnsson, A. V. Martin, M. Adolph, A. Aquila, S. Bajt, A. Barty, C. Bostedt, J. Bozek, C. Caleman, R. Coffee, N. Coppola, T. Delmas, S. Epp, B. Erk, L. Foucar, T. Gorkhover, L. Gumprecht, 
A. Hartmann, R. Hartmann, G. Hauser, P. Holl, A. Hömke, N. Kimmel, F. Krasniqi, K.-U. Kühnel, J. Maurer, M. Messerschmidt, R. Moshammer, C. Reich, B. Rudek, R. Santra, I. Schlichting, C. Schmidt, S. Schorb, J. Schulz, H. Soltau, J. C. H. Spence, D. Starodub, L. Strüder, J. Thøgersen, M. J. J. Vrakking, G. Weidenspointner, T. A. White, C. Wunderer, G. Meijer, J. Ullrich, H. Stapelfeldt, D. Rolles, and H. N. Chapman, "X-ray diffraction from isolated and strongly aligned gas-phase molecules with a free-electron laser," Phys. Rev. Lett. 112, 083002 (2014), arXiv:1307.4577 [physics]

${ }^{6}$ S. Stern, L. Holmegaard, F. Filsinger, A. Rouzée, A. Rudenko, P. Johnsson, A. V. Martin, A. Barty, C. Bostedt, J. D. Bozek, R. N. Coffee, S. Epp, B. Erk, L. Foucar, R. Hartmann, N. Kimmel, K.-U. Kühnel, J. Maurer, M. Messerschmidt, B. Rudek, D. G. Starodub, J. Thøgersen, G. Weidenspointner, T. A. White, H. Stapelfeldt, D. Rolles, H. N. Chapman, and J. Küpper, "Toward atomic resolution diffractive imaging of isolated molecules with x-ray free-electron lasers," Faraday Disc. 171, 393 (2014), arXiv:1403.2553 [physics],

${ }^{7}$ A. H. Zewail, "4D ultrafast electron diffraction, crystallography, and microscopy," Annu. Rev. Phys. Chem. 57, 65-103 (2006).

${ }^{8}$ A. A. Ischenko, P. M. Weber, and R. J. D. Miller, "Capturing chemistry in action with electrons: Realization of atomically resolved reaction dynamics," Chem. Rev. 117, 11066 11124 (2017),

${ }^{9}$ C. I. Blaga, J. Xu, A. D. DiChiara, E. Sistrunk, K. Zhang, P. Agostini, T. A. Miller, L. F. DiMauro, and C. D. Lin, "Imaging ultrafast molecular dynamics with laser-induced electron diffraction," Nature 483, 194-197 (2012).

${ }^{10}$ B. Wolter, M. G. Pullen, A. T. Le, M. Baudisch, K. Doblhoff-Dier, A. Senftleben, M. Hemmer, C. D. Schroter, J. Ullrich, T. Pfeifer, R. Moshammer, S. Gräfe, O. Vendrell, C. D. Lin, and J. Biegert, "Ultrafast electron diffraction imaging of bond breaking in di-ionized acetylene," Science 354, 308-312 (2016).

${ }^{11}$ H. Stapelfeldt, E. Constant, and P. B. Corkum, "Wave-packet structure and dynamics measured by Coulomb explosion," Phys. Rev. Lett. 74, 3780-3783 (1995)

${ }^{12}$ M. Pitzer, M. Kunitski, A. S. Johnson, T. Jahnke, H. Sann, F. Sturm, L. P. H. Schmidt, H. Schmidt-Böcking, R. Dörner, J. Stohner, J. Kiedrowski, M. Reggelin, S. Marquardt, A. Schießer, R. Berger, and M. S. Schöffler, "Direct determination of absolute molecular stereochemistry in gas phase by Coulomb explosion imaging," Science 341, 1096-1100 2013). 
${ }^{13}$ K. Nagaya, K. Motomura, E. Kukk, Y. Takahashi, K. Yamazaki, S. Ohmura, H. Fukuzawa, S. Wada, S. Mondal, T. Tachibana, Y. Ito, R. Koga, T. Sakai, K. Matsunami, K. Nakamura, M. Kanno, A. Rudenko, C. Nicolas, X. J. Liu, C. Miron, Y. Zhang, Y. Jiang, J. Chen, M. Anand, D. E. Kim, K. Tono, M. Yabashi, M. Yao, H. Kono, and K. Ueda, "Femtosecond charge and molecular dynamics of I-containing organic molecules induced by intense x-ray free-electron laser pulses," Faraday Disc. 194, 537-562 (2016),

${ }^{14}$ J. Itatani, J. Levesque, D. Zeidler, H. Niikura, H. Pépin, J. C. Kieffer, P. B. Corkum, and D. M. Villeneuve, "Tomographic imaging of molecular orbitals," Nature 432, 867-871 $(2004)$.

${ }^{15}$ C. Vozzi, M. Negro, F. Calegari, G. Sansone, M. Nisoli, S. De Silvestri, and S. Stagira, "Generalized molecular orbital tomography," Nat. Phys. 7, 822-826 (2011).

${ }^{16}$ H. J. Wörner, J. B. Bertrand, D. V. Kartashov, P. B. Corkum, and D. M. Villeneuve, "Following a chemical reaction using high-harmonic interferometry," Nature 466, 604-607 $2010)$.

${ }^{17}$ T. Baumert, B. Bühler, R. Thalweiser, and G. Gerber, "Femtosecond spectroscopy of molecular autoionization and fragmentation," Phys. Rev. Lett. 64, 733-736 (1990).

${ }^{18}$ A. Stolow and J. G. Underwood, "Time-resolved photoelectron spectroscopy of nonadiabatic dynamics in polyatomic molecules," Adv. Chem. Phys. 139, 497-584 (2008)

${ }^{19}$ R. Boll, A. Rouzée, M. Adolph, D. Anielski, A. Aquila, S. Bari, C. Bomme, C. Bostedt, J. D. Bozek, H. N. Chapman, L. Christensen, R. Coffee, N. Coppola, S. De, P. Decleva, S. W. Epp, B. Erk, F. Filsinger, L. Foucar, T. Gorkhover, L. Gumprecht, A. Hömke, L. Holmegaard, P. Johnsson, J. S. Kienitz, T. Kierspel, F. Krasniqi, K.-U. Kühnel, J. Maurer, M. Messerschmidt, R. Moshammer, N. L. M. Müller, B. Rudek, E. Savelyev, I. Schlichting, C. Schmidt, F. Scholz, S. Schorb, J. Schulz, J. Seltmann, M. Stener, S. Stern, S. Techert, J. Thøgersen, S. Trippel, J. Viefhaus, M. Vrakking, H. Stapelfeldt, J. Küpper, J. Ullrich, A. Rudenko, and D. Rolles, "Imaging molecular structure through femtosecond photoelectron diffraction on aligned and oriented gas-phase molecules," Faraday Disc. 171, $57-80(2014)$, arXiv:1407.7782 [physics]

${ }^{20}$ J. C. Williamson, J. M. Cao, H. Ihee, H. Frey, and A. H. Zewail, "Clocking transient chemical changes by ultrafast electron diffraction," Nature 386, 159-162 (1997)

${ }^{21}$ J. Yang, X. Zhu, T. J. A. Wolf, Z. Li, J. P. F. Nunes, R. Coffee, J. P. Cryan, M. Gühr, K. Hegazy, T. F. Heinz, K. Jobe, R. Li, X. Shen, T. Veccione, S. Weathersby, K. J. Wilkin, 
C. Yoneda, Q. Zheng, T. J. Martínez, M. Centurion, and X. Wang, "Imaging $\mathrm{CF}_{3} \mathrm{I}$ conical intersection and photodissociation dynamics with ultrafast electron diffraction," Science 361, 64-67 (2018).

${ }^{22}$ T. J. A. Wolf, D. M. Sanchez, J. Yang, R. M. Parrish, J. P. F. Nunes, M. Centurion, R. Coffee, J. P. Cryan, M. Gühr, K. Hegazy, A. Kirrander, R. K. Li, J. Ruddock, X. Shen, T. Vecchione, S. P. Weathersby, P. M. Weber, K. Wilkin, H. Yong, Q. Zheng, X. J. Wang, M. P. Minitti, and T. J. Martinez, "The photochemical ring-opening of 1,3-cyclohexadiene imaged by ultrafast electron diffraction," Nat. Chem. 11, 504-509 (2019).

${ }^{23}$ E. T. Karamatskos, G. Goldsztejn, S. Raabe, P. Stammer, T. Mullins, A. Trabattoni, R. R. Johansen, H. Stapelfeldt, S. Trippel, M. J. J. Vrakking, J. Küpper, and A. Rouzée, "Atomic-resolution imaging of carbonyl sulfide by laser-induced electron diffraction," J. Chem. Phys. 150, 244301 (2019), arXiv:1905.03541 [physics].

${ }^{24}$ A. Trabattoni, J. Wiese, U. De Giovannini, J.-F. Olivieri, T. Mullins, J. Onvlee, S.-K. Son, B. Frusteri, A. Rubio, S. Trippel, and J. Küpper, "Setting the photoelectron clock through molecular alignment," Nat. Commun. 11, 2546 (2020), arXiv:1802.06622 [physics],

${ }^{25}$ E. Karamatskos, Molecular-Frame Angularly-Resolved Photoelectron Spectroscopy, Dissertation, Universität Hamburg, Hamburg, Germany (2019).

${ }^{26}$ J. W. Rabalais, J. M. McDonald, V. Scherr, and S. P. McGlynn, "Electronic spectroscopy of isoelectronic molecules. II. Linear triatomic groupings containing sixteen valence electrons," Chem. Rev. 71, 73-108 (1971).

${ }^{27}$ N. Sivakumar, G. E. Hall, P. L. Houston, J. W. Hepburn, and I. Burak, "State-resolved photodissociation of OCS monomers and clusters," J. Chem. Phys. 88, 3692-3708 (1988) ${ }^{28}$ Y. Sato, Y. Matsumi, M. Kawasaki, K. Tsukiyama, and R. Bersohn, "Ion imaging of the photodissociation of OCS near 217 and 230 nm," J. Phys. Chem. 99, 16307-16314 (1995) ${ }^{29}$ T. Suzuki, H. Katayanagi, S. Nanbu, and M. Aoyagi, "Nonadiabatic bending dissociation in 16 valence electron system OCS," J. Chem. Phys. 109, 5778-5794 (1998),

${ }^{30}$ W. H. Breckenridge and H. Taube, "Ultraviolet absorption spectrum of carbonyl sulfide," J. Chem. Phys. 52, 1713-1715 (1970),

${ }^{31}$ N. Sivakumar, I. Burak, W. Y. Cheung, P. L. Houston, and J. W. Hepburn, "State-resolved photofragmentation of carbonyl sulfide (OCS) monomers and clusters," J. Phys. Chem. 89. $3609-3611(1985)$ 
${ }^{32}$ H. Katayanagi, Y. Mo, and T. Suzuki, "223 nm photodissociation of OCS. Two components in $\mathrm{S}\left({ }^{1} D_{2}\right)$ and $\mathrm{S}\left({ }^{3} P_{2}\right)$ channels," Chem. Phys. Lett. 247, 571-576 (1995)

${ }^{33}$ Z. H. Kim, A. J. Alexander, and R. N. Zare, "Speed-dependent photofragment orientation in the photodissociation of OCS at 223 nm," J. Phys. Chem. A 103, 10144-10148 (1999) ${ }^{34}$ A. Sugita, M. Mashino, M. Kawasaki, Y. Matsumi, R. Bersohn, G. Trott-Kriegeskorte, and K. a.-H. Gericke, "Effect of molecular bending on the photodissociation of OCS," J. Chem. Phys. 112, 7095-7101 (2000)

${ }^{35}$ T. P. Rakitzis, P. C. Samartzis, and T. N. Kitsopoulos, "Observing the symmetry breaking in the angular distributions of oriented photofragments using velocity mapping," J. Chem. Phys. 111, 10415-10417 (1999),

${ }^{36}$ H. Katayanagi and T. Suzuki, "Non-adiabatic bending dissociation of OCS: the effect of bending excitation on the transition probability," Chem. Phys. Lett. 360, 104-110 (2002).

${ }^{37}$ A. M. Rijs, E. H. G. Backus, C. A. de Lange, M. H. M. Janssen, N. P. C. Westwood, K. Wang, and V. McKoy, "Rotationally resolved photoionization dynamics of hot CO fragmented from OCS," J. Chem. Phys. 116, 2776-2782 (2002).

${ }^{38}$ W. Wei, C. J. Wallace, G. C. McBane, and S. W. North, "Photodissociation dynamics of OCS near $214 \mathrm{~nm}$ using ion imaging," J. Chem. Phys. 145, 024310 (2016).

${ }^{39}$ M. Brouard, A. V. Green, F. Quadrini, and C. Vallance, "Photodissociation dynamics of OCS at $248 \mathrm{~nm}$ : The $\mathrm{S}\left({ }^{1} D_{2}\right)$ atomic angular momentum polarization," J. Chem. Phys. 127. 084304 (2007)

${ }^{40}$ A. J. van den Brom, T. P. Rakitzis, and M. H. M. Janssen, "Photodissociation of laboratory oriented molecules: Revealing molecular frame properties of nonaxial recoil," J. Chem. Phys. 121, 11645-11652 (2004),

${ }^{41}$ A. J. van den Brom, T. P. Rakitzis, and M. H. M. Janssen, "State-to-state photodissociation of carbonyl sulfide $\left(v_{2}=0,1 \mid J I M\right)$. II. The effect of initial bending on coherence of $\mathrm{S}\left({ }^{1} D_{2}\right)$ polarization," J. Chem. Phys. 123, 164313 (2005).

${ }^{42}$ T. P. Rakitzis, A. J. van den Brom, and M. H. M. Janssen, "Directional dynamics in the photodissociation of oriented molecules," Science 303, 1852-1854 (2004).

${ }^{43}$ M. L. Lipciuc and M. H. M. Janssen, "Slice imaging of quantum state-to-state photodissociation dynamics of OCS," Phys. Chem. Chem. Phys. 8, 3007-3016 (2006).

${ }^{44}$ M. L. Lipciuc, T. P. Rakitzis, W. L. Meerts, G. C. Groenenboom, and M. H. M. Janssen, "Towards the complete experiment: measurement of $\mathrm{S}\left({ }^{1} D_{2}\right)$ polarization in correlation with 
single rotational states of $\mathrm{CO}(J)$ from the photodissociation of oriented $\operatorname{OCS}\left(v_{2}=1 \mid J l M=\right.$ 111)," Phys. Chem. Chem. Phys. 13, 8549-8559 (2011).

${ }^{45}$ D. Sofikitis, J. Suarez, J. A. Schmidt, T. P. Rakitzis, S. C. Farantos, and M. H. M. Janssen,

"Recoil inversion in the photodissociation of carbonyl sulfide near $234 \mathrm{~nm}$," Phys. Rev. Lett. 118, 253001 (2017).

${ }^{46}$ D. Sofikitis, J. Suarez, J. A. Schmidt, T. P. Rakitzis, S. C. Farantos, and M. H. M. Janssen, "Exit-channel recoil resonances by imaging the photodissociation of single quantum-stateselected OCS molecules," Phys. Rev. A 98, 033417 (2018).

${ }^{47}$ J. A. Schmidt, M. S. Johnson, G. C. McBane, and R. Schinke, "The ultraviolet spectrum of OCS from first principles: Electronic transitions, vibrational structure and temperature dependence," J. Chem. Phys. 137, 054313 (2012)

${ }^{48}$ J. A. Schmidt, M. S. Johnson, S. Hattori, N. Yoshida, S. Nanbu, and R. Schinke, "OCS photolytic isotope effects from first principles: sulfur and carbon isotopes, temperature dependence and implications for the stratosphere," Atmos. Chem. Phys. 13, 1511-1520 (2013).

${ }^{49}$ J. A. Schmidt, M. S. Johnson, G. C. McBane, and R. Schinke, "Multi-state analysis of the OCS ultraviolet absorption including vibrational structure," J. Chem. Phys. 136, 131101 $(2012)$.

${ }^{50}$ G. C. McBane, J. A. Schmidt, M. S. Johnson, and R. Schinke, "Ultraviolet photodissociation of OCS: Product energy and angular distributions," J. Chem. Phys. 138, 094314 (2013).

${ }^{51}$ S. O. Danielache, S. Nanbu, C. Eskebjerg, M. S. Johnson, and N. Yoshida, "Carbonyl sulfide isotopologues: Ultraviolet absorption cross sections and stratospheric photolysis," J Chem. Phys. 131, 024307 (2009)

${ }^{52}$ C. Brühl, J. Lelieveld, P. J. Crutzen, and H. Tost, "The role of carbonyl sulphide as a source of stratospheric sulphate aerosol and its impact on climate," Atmos. Chem. Phys.

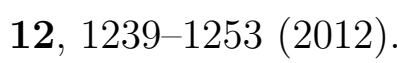

${ }^{53}$ S. Hattori, K. Kamezaki, and N. Yoshida, "Constraining the atmospheric OCS budget from sulfur isotopes," PNAS 117, 20447-20452 (2020).

${ }^{54}$ M. L. Lipciuc and M. H. M. Janssen, "Slice imaging of the quantum state-to-state cross section for photodissociation of state-selected rovibrational bending states of OCS $\left(v_{2}=\right.$ $0,1,2 \mid J l M)+h \nu \rightarrow \mathrm{CO}(J)+\mathrm{S}\left({ }^{1} D_{2}\right)$, J. Chem. Phys. 126, $194318(2007)$ 
${ }^{55} \mathrm{~B}$. W. Toulson and C. Murray, "Decomposing the first absorption band of OCS using photofragment excitation spectroscopy," J. Phys. Chem. A 120, 6745-6752 (2016).

${ }^{56}$ G. Nan, I. Burak, and P. Houston, "Photodissociation of OCS at $222 \mathrm{~nm}$. The triplet channel," Chem. Phys. Lett. 209, 383 - 389 (1993).

${ }^{57}$ Y. Mo, H. Katayanagi, M. C. Heaven, and T. Suzuki, "Simultaneous measurement of recoil velocity and alignment of $S\left({ }^{1} D_{2}\right)$ atoms in photodissociation of OCS," Phys. Rev. Lett. 77, 830-833 (1996).

${ }^{58}$ T. P. Rakitzis, P. C. Samartzis, and T. N. Kitsopoulos, "Complete measurement of S $\left({ }^{1} D_{2}\right)$ photofragment alignment from Abel-invertible ion images," Phys. Rev. Lett. 87, 123001 (2001).

${ }^{59}$ S. K. Lee, R. Silva, S. Thamanna, O. S. Vasyutinskii, and A. G. Suits, "S $\left({ }^{1} D_{2}\right)$ atomic orbital polarization in the photodissociation of OCS at $193 \mathrm{~nm}$ : Construction of the complete density matrix," J. Chem. Phys. 125, 144318 (2006)

${ }^{60}$ J. A. Schmidt and J. M. H. Olsen, "Photodissociation of OCS: Deviations between theory and experiment, and the importance of higher order correlation effects," J. Chem. Phys.

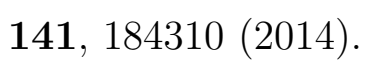

${ }^{61}$ E. T. Karamatskos, S. Raabe, T. Mullins, A. Trabattoni, P. Stammer, G. Goldsztejn, R. R. Johansen, K. Długołęcki, H. Stapelfeldt, M. J. J. Vrakking, S. Trippel, A. Rouzée, and J. Küpper, "Molecular movie of ultrafast coherent rotational dynamics of OCS," Nat. Commun. 10, 3364 (2019), arXiv:1807.01034 [physics]

${ }^{62}$ J. Wiese, J. Onvlee, S. Trippel, and J. Küpper, "Strong-field ionization of complex molecules," (2020), under review, arXiv:2003.02116 [physics].

${ }^{63}$ M. Hillenkamp, S. Keinan, and U. Even, "Condensation limited cooling in supersonic expansions," J. Chem. Phys. 118, 8699-8705 (2003)

${ }^{64}$ V. Dribinski, A. Ossadtchi, V. A. Mandelshtam, and H. Reisler, "Reconstruction of Abeltransformable images: The Gaussian basis-set expansion Abel transform method," Rev Sci. Instrum. 73, 2634 (2002)

${ }^{65}$ H.-D. Meyer, U. Manthe, and L. Cederbaum, "The multi-configurational time-dependent Hartree approach," Chem. Phys. Lett. 165, 73-78 (1990).

${ }^{66}$ U. Manthe, H. D. Meyer, and L. S. Cederbaum, "Wave-packet dynamics within the multiconfiguration Hartree framework: General aspects and application to NOCl," J. Phys. Chem. 97, 3199-3213 (1992). 
${ }^{67}$ R. Welsch, F. Huarte-Larrañaga, and U. Manthe, "State-to-state reaction probabilities within the quantum transition state framework," J. Chem. Phys. 136, 064117 (2012).

${ }^{68} \mathrm{R}$. Welsch and U. Manthe, "Thermal flux based analysis of state-to-state reaction probabilities," Mol. Phys. 110, 703-715 (2012).

${ }^{69} \mathrm{U}$. Manthe, "A time-dependent discrete variable representation for (multiconfiguration) Hartree methods," J. Chem. Phys. 105, 6989-6994 (1996).

${ }^{70} \mathrm{U}$. Manthe, "On the integration of the multi-configurational time-dependent Hartree (MCTDH) equations of motion," Chem. Phys. 329, 168-178 (2006).

${ }^{71}$ S. Morse, M. Takahashi, J. H. D. Eland, and L. Karlsson, "Dissociative photoionisation of OCS from threshold to 40.8 eV," Int. J. Mass Spectrom. Ion Processes 184, 67-74 (1999)

${ }^{72}$ H. Stapelfeldt, E. Constant, H. Sakai, and P. B. Corkum, "Time-resolved Coulomb explosion imaging: A method to measure structure and dynamics of molecular nuclear wave packets," Phys. Rev. A 58, 426-433 (1998).

${ }^{73}$ M. V. Ammosov, N. B. Delone, and V. P. Krainov, "Tunnel ionization of complex atoms and of atomic ions in an alternating electromagnetic field," Soviet Physics - JETP 64. 1191-1194 (1986)

${ }^{74}$ I. Wilkinson, A. E. Boguslavskiy, J. Mikosch, J. B. Bertrand, H. J. Wörner, D. M. Villeneuve, M. Spanner, S. Patchkovskii, and A. Stolow, "Excited state dynamics in $\mathrm{SO}_{2}$. i. bound state relaxation studied by time-resolved photoelectron-photoion coincidence spectroscopy," J. Chem. Phys. 140, 204301 (2014).

${ }^{75}$ S. Mai, P. Marquetand, and L. González, "Non-adiabatic and intersystem crossing dynamics in $\mathrm{SO}_{2}$. ii. the role of triplet states in the bound state dynamics studied by surface-hopping simulations," J. Chem. Phys. 140, 204302 (2014)

${ }^{76}$ A. Owens, A. Yachmenev, S. N. Yurchenko, and J. Küpper, "Climbing the Rotational Ladder to Chirality," Phys. Rev. Lett. 121, 193201 (2018), arXiv:1802.07803 [physics]

${ }^{77}$ S. Pathak, L. M. Ibele, R. Boll, C. Callegari, A. Demidovich, B. Erk, R. Feifel, R. Forbes, M. Di Fraia, L. Giannessi, C. S. Hansen, D. M. P. Holland, R. A. Ingle, R. Mason, O. Plekan, K. C. Prince, A. Rouzee, R. J. Squibb, J. Tross, M. N. R. Ashfold, B. F. E. Curchod, and D. Rolles, "Tracking the ultraviolet-induced photochemistry of thiophenone during and after ultrafast ring opening," Nat. Chem. 12, 795 (2020). 